\title{
Proliferating Myeloid Cell to Total Cell Ratio Measurement
}

National Cancer Institute

\section{Source}

National Cancer Institute. Proliferating Myeloid Cell to Total Cell Ratio Measurement. NCI Thesaurus. Code C127634.

The determination of the ratio of proliferating myeloid cells compared to total cells present in a sample. The measurement may be expressed as a ratio or percentage. 